\title{
Tailoring the message with selective reporting
}

\author{
Magnus Løberg ${ }^{1,2}$ (D) Mette Kalager ${ }^{1,2,3} \cdot$ Geir Hoff $^{4,5}$
}

Received: 15 May 2018/ Accepted: 25 May 2018/Published online: 30 May 2018

(C) Springer Science+Business Media B.V., part of Springer Nature 2018

We have read the article by Niedermaier et al. [1] with great interest. This is a modelling study aiming to estimate the sensitivity and specificity of a combined strategy of sigmoidoscopy and fecal immunochemical test (FIT) screening to detect colorectal neoplasia. To inform the model, the authors performed a meta-analysis of studies that have evaluated the site-specific FIT sensitivity, with colonoscopy as the gold standard.

An obvious limitation with sigmoidoscopy screening is the low sensitivity in the proximal colon, both for early detection of cancer, and for detection and removal of adenomas. A way to mitigate this limitation, could be to add a FIT to the sigmoidoscopy screening, and thus try to increase the detection rate of proximal cancers and adenomas through increased referral to follow-up colonoscopy.

The idea described above is not new: In the Norwegian Colorectal Cancer Prevention (NORCCAP) trial [2], individuals in the screening arm were randomized to screening with sigmoidoscopy or a combination of sigmoidoscopy and a once-only FIT (combined strategy). Surprisingly, the combined strategy was less successful than anticipated, even though attendance rate was comparable in the two groups (combined strategy 61\%, sigmoidoscopy only 65\%). During screening and follow-up colonoscopy, there were no differences in detection of any adenoma, advanced adenoma or cancer between the groups. More importantly,

Magnus Løberg

magnus.loberg@medisin.uio.no

1 Clinical Effectiveness Research Group, Institute of Health and Society, University of Oslo, Postbox 1089, 0318 Blindern, Oslo, Norway

2 Department of Transplantation Medicine and KG Jebsen Colorectal Cancer Research Center, Oslo University Hospital, Oslo, Norway

3 Department of Epidemiology, Harvard TH Chan School of Public Health, Boston, MA, USA

4 Telemark Hospital, Skien, Norway

5 Cancer Registry of Norway, Oslo, Norway however, there was no difference in the long-term screening effect between the two groups: The incidence of colorectal cancer was most reduced in the sigmoidoscopy only group, while the mortality of colorectal cancer was most reduced in the combined strategy group.

We find it worrying that the authors selectively have referred to the NORCCAP findings supporting their view. They have ignored to mention that there was no difference between the groups in the detection rates of any adenoma, advanced adenoma and cancer at screening, and that the intention-to-screen analysis showed that colorectal cancer incidence was reduced in the sigmoidoscopy only group, but not in the combined strategy group.

We are surprised to see that Niedermaier et al. put more faith in their model than a large, well-designed randomized controlled trial with sufficient follow-up to capture patient important outcomes, such as colorectal cancer incidence and mortality. This is in stark contrast to the GRADE system [3], where evidence from randomized trials are ranked above observational studies, and where models are typically considered as low-quality evidence.

\section{References}

1. Niedermaier T, Weigl K, Hoffmeister M, Brenner H. Diagnostic performance of flexible sigmoidoscopy combined with fecal immunochemical test in colorectal cancer screening: meta-analysis and modeling. Eur J Epidemiol. 2017;32(6):481-93.

2. Holme $\mathrm{O}$, Loberg $\mathrm{M}$, Kalager $\mathrm{M}$, et al. Effect of flexible sigmoidoscopy screening on colorectal cancer incidence and mortality: a randomized clinical trial. JAMA. 2014;312:606-15.

3. Guyatt GH, Oxman AD, Vist GE, Kunz R, Falck-Ytter Y, AlonsoCoello P, et al. GRADE: an emerging consensus on rating quality of evidence and strength of recommendations. BMJ. 2008;336(7650):924-6. 